\title{
A Re-testing Range is Recommended for 13C- and 14C-urea Breath Tests for Helicobacter Pylori Infection in China
}

\section{Xiangyu Wang}

Shenzhen University First Affiliated Hospital: Shenzhen Second People's Hospital

https://orcid.org/0000-0003-2596-8483

\section{Shuzhen Zhang}

Shenzhen Kuichong People's Hospital

\section{Eng Guan Chua}

University of Western Australia

\section{Yongsheng He}

Shenzhen Kuichong People's Hospital

\section{Xiaofeng Li}

Shenzhen People's Hospital

\section{Aijun Liu}

Shenzhen Kuichong People's Hospital

\section{Haiting Chen}

Shenzhen Kuichong People's Hospital

\section{Michael J. Wise}

The University of Western Australia

\section{Barry J. Marshall}

The University of Western Australia

\section{Dayong Sun}

Shenzhen University First Affiliated Hospital: Shenzhen Second People's Hospital

\section{Xuehong Li}

Shenzhen Kuichong People's Hospital

Alfred Chin Yen Tay ( $\nabla$ alfred.tay@uwa.edu.au )

University of Western Australia https://orcid.org/0000-0001-9705-4010

\section{Research}

Keywords: Helicobacter pylori, urea breath test, diagnostic performance, indeterminate range 
DOl: https://doi.org/10.21203/rs.3.rs-356067/v1

License: (c) (1) This work is licensed under a Creative Commons Attribution 4.0 International License. Read Full License 


\section{A re-testing range is recommended for ${ }^{13} \mathrm{C}$ - and ${ }^{14} \mathrm{C}$-urea 2 breath tests for Helicobacter pylori infection in China}

3 Xiangyu Wang ${ }^{1,2 *}$, Shuzhen Zhang ${ }^{2 *}$, Eng Guan $\mathrm{Chua}^{3}$, Yongsheng $\mathrm{He}^{4}$, Xiaofeng $\mathrm{Li}^{5}$, Aijun

$4 \mathrm{Liu}^{4}$, Haiting Chen ${ }^{2}$, Michael J. Wise ${ }^{3,6}$, Barry J. Marshall ${ }^{3}$, Dayong $\mathrm{Sun}^{1}$, Xuehong $\mathrm{Li}^{2 \#}$, and 5 Chin Yen Tay ${ }^{3 \#}$

$6{ }^{1}$ Department of Gastroenterology, Shenzhen Second People's Hospital/ the First Affiliated

7 Hospital of Shenzhen University, Health Science Center, Shenzhen 518035, China.

$8 \quad{ }^{2}$ Department of Clinical Laboratory, Kuichong People's Hospital, Shenzhen 518116, China.

$9 \quad{ }^{3}$ The Marshall Centre for Infectious Diseases Research and Training, University of Western

10 Australia, Perth 6009, Australia.

$11{ }^{4}$ Department of Gastroenterology, Kuichong People's Hospital, Shenzhen 518116, China.

$12{ }^{5}$ Department of Nuclear Medicine, Shenzhen People's Hospital, Shenzhen 518001, China.

$13{ }^{6}$ Department of Computer Science and Software Engineering, University of Western Australia,

14 Perth 6009, Australia.

$15 *$ Both Xiangyu Wang and Shuzhen Zhang contributed equally to this work.

16 \# Corresponding authors:

17 Dr. Chin Yen Tay ${ }^{3}$ (Email: alfred.tay@uwa.edu.au); Dr. Xuehong Li² (Email:

18 lihongsz@163.com). 


\section{Abstract}

20 Background: The urea breath test (UBT) is widely used for diagnosing Helicobacter pylori

21 infection. In our hospital, some UBT findings were contradictory to the histology outcomes,

22 therefore this study aimed to assess and compare the diagnostic performance of both ${ }^{13} \mathrm{C}$ - and

$23 \quad{ }^{14}$ C-UBT assays.

24 Methods: We recruited 484 H. pylori-treatment naïve patients, among which 217 and 267 were

25 tested by the ${ }^{13} \mathrm{C}$-UBT or ${ }^{14} \mathrm{C}$-UBT, respectively. The cutoff value for $H$. pylori positivity based

26 on manufacturer's instruction was $4 \%$ DOB for the ${ }^{13} \mathrm{C}$-UBT, and $100 \mathrm{DPM}$ for the ${ }^{14} \mathrm{C}-\mathrm{UBT}$.

27 Gastric biopsies of the antrum and corpus were obtained during endoscopy for histopathology.

28 Results: In patients who were tested using the ${ }^{13} \mathrm{C}$-UBT kit, histopathology was positive in 136

29 out of 164 UBT-positive patients ( $82.9 \%$ concordance), and negative in 46 out of 53 UBT-

30 negative cases (86.8\% concordance). For the ${ }^{14} \mathrm{C}$-UBT-tested patients, histopathology was

31 positive for $H$. pylori in 186 out of 220 UBT-positive patients ( $84.5 \%$ concordance), and negative

32 in 41 out of 47 UBT-negative cases ( $87.2 \%$ concordance). While the ${ }^{13} \mathrm{C}$-UBT and ${ }^{14} \mathrm{C}$-UBT each

33 had a high sensitivity level of $95.1 \%$ and $96.9 \%$, respectively, their specificity was low, at $62.2 \%$

34 and 54.7\%, respectively. By using new optimal cutoff values and including an indeterminate

35 range (3-10.3\% DOB for ${ }^{13} \mathrm{C}-\mathrm{UBT}$ and $87-237 \mathrm{DPM}$ for $\left.{ }^{14} \mathrm{C}-\mathrm{UBT}\right)$, the specificity values can be

36 improved to $76.7 \%$ and $76.9 \%$ for the ${ }^{13} \mathrm{C}$ - and ${ }^{14} \mathrm{C}-\mathrm{UBT}$, respectively. 
38 Conclusions: The establishment of an indeterminate range is recommended to allow for repeated

39 testing to confirm $H$. pylori infection, and thereby avoiding unnecessary antibiotic treatment.

40 Trial registration: Chinese Clinical Trial Registry, ChiCTR2000041570. Registered 29 December

41 2020- Retrospectively registered, http://www.chictr.org.cn/edit.aspx?pid=66416\&htm=4

42 (263/350 words)

43

44 Key words: Helicobacter pylori, urea breath test, diagnostic performance, indeterminate

45 range 


\section{Introduction}

47 Helicobacter pylori infection is common in China, with an overall estimated prevalence of $55.8 \%$

48 (1). It is an important gastric pathogen that can lead to several gastroduodenal disorders including

49 chronic gastritis, gastric atrophy and peptic ulcer disease, and less commonly, to gastric

50 adenocarcinoma and mucosa associated lymphoid tissue (MALT) lymphoma $(2,3)$.

51 Helicobacter pylori is able to convert urea into carbon dioxide and ammonia via its urease

52 enzyme, where the ammonia is used to neutralize the acid for its survival in the stomach (4).

53 Based on this feature of $H$. pylori, the urea breath test (UBT), a non-invasive $H$. pylori infection

54 diagnostic method was developed. This requires a patient to swallow a capsule containing a dose

55 of urea labeled with carbon-13 $\left({ }^{13} \mathrm{C}\right)$ or carbon-14 $\left({ }^{14} \mathrm{C}\right)$ isotope. If the patient is an $H$. pylori

56 carrier, the labeled urea will be hydrolyzed by the bacterial urease enzyme within the stomach,

57 resulting in the release of labeled carbon dioxide which is then absorbed into the bloodstream and

58 expelled from the lungs in a few minutes. Hence the amount of labeled carbon dioxide within a

59 patient's breath sample can be measured to determine current $H$. pylori infection status $(5,6)$.

60 Due to its accuracy, simplicity and non-invasive nature, the UBT has been the preferred method

61 of many medical professionals for testing $H$. pylori infection in their patients. Both the ${ }^{13} \mathrm{C}-\mathrm{UBT}$

62 and the ${ }^{14} \mathrm{C}$-UBT are widely used. The former utilizes the stable ${ }^{13} \mathrm{C}$ isotope of carbon while the

63 latter uses the radioactive ${ }^{14} \mathrm{C}$ carbon isotope. Nevertheless, it is important to mention that both

64 are naturally occurring isotopes and the radiation exposure from the ${ }^{14} \mathrm{C}-\mathrm{UBT}$ is even lower than

65 that from background radiation (7). In fact, the ${ }^{14} \mathrm{C}-\mathrm{UBT}$ has been approved by the Food and 
66 Drug Administration (FDA) of the United States for its usage in everyone, including children and

67 pregnant women (8).

68 While both UBT are useful in detecting $H$. pylori infection, we noticed that several UBT results

69 were contradictory to the outcomes determined via histopathology examination, prompting us to

70 reconsider the diagnostic accuracy of the commercial UBT kits used for screening $H$. pylori

71 infection in our hospital. In this study, we recruited 484 individuals who underwent endoscopic

72 examination at Shenzhen Kuichong People's Hospital, among which 217 and 267 were tested for

73 H. pylori infection using the ${ }^{13} \mathrm{C}$-UBT and ${ }^{14} \mathrm{C}$-UBT, respectively. By comparing the outcomes to

74 that of histopathology examination of gastric biopsies, which is the gold standard for diagnosing

75 H. pylori infection, we assessed the diagnostic performance of both UBT kits. Additionally, as

76 these commercial kits available for use at our hospital provide only a cutoff value for $H$. pylori

77 positivity, we therefore sought to determine a value range that indicates an indeterminate

78 outcome requiring a repeat UBT, below which we can with confidence infer the absence of an

79 infection.

80

81 Material and Methods

82 Overview of entire study

83 The schematic flow of experimental program was shown in Figure 1. 


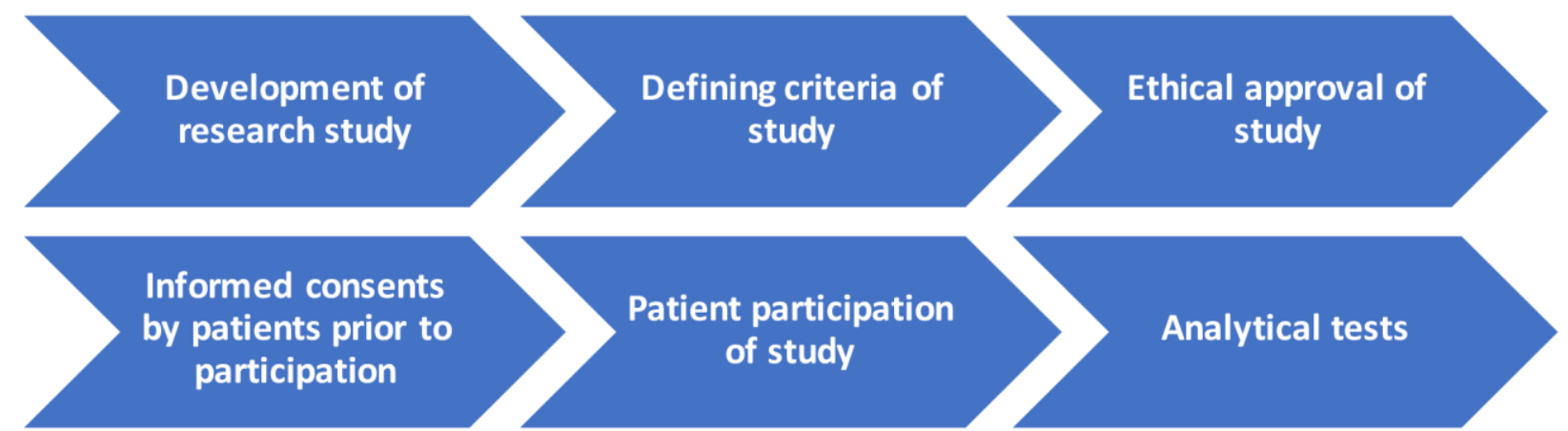

91 Figure 1. The overview of entire study.

\section{Development of research study}

94 During the clinical practice, we noticed that several UBT results were contradictory to the

95 outcomes determined via histopathology examination, prompting us to reassess the diagnostic

96 performance of both ${ }^{13} \mathrm{C}$-UBT and ${ }^{14} \mathrm{C}$-UBT commercial kits used for screening $H$. pylori

97 infection in our hospital.

99 Defining the criteria of study

100 The following exclusion criteria on our study subjects were applied: (i) previous treatment of $H$.

101 pylori infection, (ii) received proton pump inhibitor, $\mathrm{H}_{2}$ receptor antagonist, expectorant, or

102 antibiotic treatment within the last four weeks prior to endoscopy, (iii) history of gastric surgery,

103 (iv) diagnosed with gastrointestinal cancer, (v) had severe heart, lung, liver, kidney or blood

104 system disorder, (vi) aged below 18 or above 70 years old and (vii) pregnancy. 
107 This study was approved by the research ethics committee of Shenzhen Kuichong People's

108 Hospital (reference no. 201609) and registered at www.chictr.org.cn (reference no.

109 ChiCTR2000041570). Each patient was given a detailed introduction to the purpose and process

110 of the research by a gastroenterologist. Written and informed consents were obtained from all

111 patients prior to their participation in the study.

112

\section{Study population}

114 From January 2017 to November 2018, following the application of the exclusion criteria, 484

115 patients (18-70 years of age) who visited Shenzhen Kuichong People's Hospital (26 Kuixin N

116 Road, Dapeng New District, Shenzhen City, China) for endoscopic check-up agreed to participate

117 in this study.

118 Three gastroenterologists were involved in this study. Prior to the endoscopy session, the patients

119 received either a ${ }^{13} \mathrm{C}-\mathrm{UBT}$ or a ${ }^{14} \mathrm{C}-\mathrm{UBT}$ at the discretion of a gastroenterologist via the alternating

120 assignment method. The general health information of patients was collected by clinical nurses.

121 During endoscopy, two gastric biopsy specimens (one each from antrum and corpus) were collected

122 for histopathology examination. The different set of people did the examination and analysed the

123 results. The testing was done by the clinical technicians without any implied or actual plan to check

124 the outcome or accuracy of the UBT. 


\section{Sample size determination}

127 The samples size required for this study was estimated based on a 95\% confidence interval using 128 the following formula (9):

130 Sample size (n1) based on sensitivity $=\frac{\mathrm{z}_{1-\frac{\alpha}{2}}^{2} \operatorname{Sens}(1-\text { Sens })}{d^{2} \text { Prev }}$

132 Sample size (n2) based on specificity $=\frac{\mathrm{z}_{1-\frac{\alpha}{2}}^{2} \operatorname{Spec}(1-\operatorname{Spec})}{d^{2}(1-\text { Prev })}$

134 Where $\mathrm{Z}$, the normal distribution value, was set to 1.96 as corresponding with the $95 \%$

135 confidence interval, and $d$, the maximum acceptable width of the $95 \%$ confidence interval, was

136 set to $10 \%$. Based on a previous study, the UBT was shown to achieve a sensitivity value of $96 \%$

137 and a specificity value of $93 \%$ (10). The prevalence rate (Prev) of $H$. pylori in Shenzhen, China

138 was $35.85 \%$ (11). Therefore, this study should recruit at least $41 \mathrm{H}$. pylori-positive patients (n1)

139 and 39 H. pylori-negative patients (n2), yielding a total sample size of 80 participants for each

$140 \quad$ UBT test.

\section{$142 \quad$ Urea breath test}

$143 \quad{ }^{13} C-U B T$

144 The ${ }^{13}$ C-UBT (Beijing Boran Pharmaceutical Co. Ltd., China) was performed according to the 145 manufacturer's instructions. Briefly, an initial baseline breath sample was collected from each 
146 patient after fasting for at least four hours prior to ingesting a capsule containing $50 \mathrm{mg}{ }^{13} \mathrm{C}$

147 isotope labeled urea with $80-100 \mathrm{~mL}$ of water. After 30 minutes of sitting, exhaled breath was

148 again collected. The ${ }^{13} \mathrm{CO}_{2}$ content within the initial and 30-min expiratory air bags were

149 analyzed using an HG-IRIS13C infrared spectrometer (Beijing Richen-Force Science \&

150 Technology Co., China). Following 30 minutes of administration, a delta over baseline (DOB)

151 value of $4 \%$ or above was regarded as a positive indicator of $H$. pylori infection.

$152{ }^{14} C-U B T$

153 The ${ }^{14}$ C-UBT (Zhonghe Headway Bio-Sci \& Tech Co. Ltd., China) was performed according to

154 the manufacturer's instruction. Briefly, patients who fasted for at least four hours were requested

155 to ingest a gelatin capsule containing $0.75 \mu \mathrm{Ci}$ of ${ }^{14} \mathrm{C}$ isotope with $20 \mathrm{~mL}$ of water. After 25

156 minutes, each patient was then asked to exhale continuously into a bottle until the purple-colored

$157 \mathrm{CO}_{2}$ capturing liquid within turned colorless. The scintillation fluid was subsequently added, and

158 the homogenized solution was measured for ${ }^{14} \mathrm{CO}_{2}$ quantity. A reading with more than 100

159 disintegrations per minute (DPM) was classified as H. pylori positive.

160

161 Histopathology

162 Two gastric biopsy specimens (one each from antrum and corpus) were sent to Da'an Clinical

163 Laboratory (Guangzhou, China) for histopathology examination. The histopathologists were

164 unaware of the UBT results. The presence of Helicobacter-like organism was confirmed with

165 routine hematoxylin and eosin (HE) staining. Giemsa staining was further performed if $\mathrm{HE}$ 
166 couldn't confirm the H. pylori clearly and in those patients with obvious inflammatory reaction,

167 but no $H$. pylori found in HE staining.

168

\section{Statistical analysis}

170 The sensitivity and specificity values of each UBT method were reported according to

171 manufacturer's recommended cut-off value. To evaluate the diagnostic capacity of each UBT

172 method, the receiver operating characteristic (ROC) curve was generated by plotting the true-

173 positive rate on the $y$ axis against the true-negative rate on the $\mathrm{x}$ axis (12). Our ROC analysis was

174 performed using the R package pROC (version 1.16.2) (13). The area under the curve (AUC) was

175 calculated to quantify the overall accuracy of each UBT method to diagnose $H$. pylori infection

176 outcomes. The optimal cutoff UBT value that generates the highest true positive rate together

177 with the lowest false positive rate, was determined by using maximum Youden index method,

178 where Youden index $=$ sensitivity + specificity -1 (14). For the comparison of categorical

179 variables, the Fisher's exact test was used. The level of statistical significance was considered at $180 \quad p<0.05$.

\section{Results}

183 Diagnostic performance of ${ }^{13} \mathrm{C}$-UBT and ${ }^{14} \mathrm{C}$-UBT with the manufacturer's recommended

184 cutoff for UBT 
185 The UBT readings, histological findings of $H$. pylori in gastric biopsies and patient demographics

186 including age and sex are available in Table S1. Among the 484 patients recruited in this study,

187217 and 267 were tested using the ${ }^{13} \mathrm{C}$ - and ${ }^{14} \mathrm{C}$-UBT kits, respectively. The numbers of $H$.

188 pylori-positive and -negative patients were $164(75.6 \%, 164 / 217)$ and $53(24.4 \%, 53 / 217)$, using

189 the ${ }^{13} \mathrm{C}-\mathrm{UBT}$, and $220(82.4 \%, 220 / 267)$ and $47(17.6 \%, 47 / 267)$, as indicated by the ${ }^{14} \mathrm{C}-\mathrm{UBT}$.

190 We next assessed the diagnostic performance of both UBT assays (Table 1). While the ${ }^{13} \mathrm{C}-\mathrm{UBT}$

191 and ${ }^{14} \mathrm{C}-\mathrm{UBT}$ each had a high sensitivity of 95.1\% (CI:89.8\%-97.8\%) and 96.9\% (CI:93.0\%-

$19298.7 \%$ ), respectively, their specificity was unsatisfactory, at 62.2\% (CI:50.1\%-73.0\%) and 54.7\%

193 (CI:42.8\%-66.1\%), respectively.

194

195 Table 1. Diagnostic performance of the ${ }^{13} \mathrm{C}-\mathrm{UBT}$ and ${ }^{14} \mathrm{C}-\mathrm{UBT}$.

\begin{tabular}{|c|c|c|c|c|c|c|c|c|}
\hline \multirow[t]{2}{*}{ Method } & \multicolumn{3}{|c|}{ Histology } & \multirow{2}{*}{$\begin{array}{c}\text { Sens (\%) } \\
95 \% \mathrm{Cl}\end{array}$} & \multirow{2}{*}{$\begin{array}{c}\text { Spec (\%) } \\
95 \% \mathrm{Cl}\end{array}$} & \multirow{2}{*}{$\begin{array}{l}\text { Acc (\%) } \\
95 \% \mathrm{Cl}\end{array}$} & \multirow{2}{*}{$\begin{array}{l}\text { FPR (\%) } \\
95 \% \mathrm{Cl}\end{array}$} & \multirow{2}{*}{$\begin{array}{c}\text { FNR (\%) } \\
95 \% \mathrm{Cl}\end{array}$} \\
\hline & & + & - & & & & & \\
\hline \multirow[t]{2}{*}{${ }^{13} \mathrm{C}$-UBT } & + & 136 & 28 & 95.1 & 62.2 & 83.9 & 37.8 & 4.9 \\
\hline & - & 7 & 46 & $89.8-97.8$ & $50.1-73.0$ & $78.4-88.2$ & $27.0-49.9$ & $2.2-10.2$ \\
\hline \multirow[t]{2}{*}{${ }^{14} \mathrm{C}$-UBT } & + & 186 & 34 & 96.9 & 54.7 & 85 & 45.3 & 3.1 \\
\hline & - & 6 & 41 & $93.0-98.7$ & $42.8-66.1$ & $80.2-88.8$ & $33.9-57.2$ & $1.3-7.0$ \\
\hline
\end{tabular}

196 UBT: urea breath test; Sens: sensitivity; Spec: specificity; Acc: accuracy; FPR: false positive 197 rate; FNR: false negative rate; CI: confidence interval.

199 We also compared the discordance of $H$. pylori infection status as determined by each UBT assay

200 to histopathology in three different age groups (Table 2). Interestingly, in patients aged 18-30

201 years, there was a significantly higher discordance between the ${ }^{13} \mathrm{C}$-UBT and histopathology 
202 outcomes as compared to the ${ }^{14} \mathrm{C}$-UBT counterparts $(29.4 \%$ versus $8.3 \%, P=0.032)$. On the other

203 hand, in patients aged above 50 years, the discordance was significantly greater in the ${ }^{14}$ C-UBT

204 group than those who were tested by the ${ }^{13} \mathrm{C}$-UBT $(28.4 \%$ versus $12.2 \%, P=0.045)$. No

205 significant difference was observed in the 31-50 years patient group.

206

207 Table 2. Discordance between UBT and histopathology findings among different patient age 208 groups.

\begin{tabular}{lccc|ccc|c}
\hline \multirow{2}{*}{ Age (years) } & \multicolumn{3}{c|}{${ }^{13}$ C-UBT } & \multicolumn{3}{c|}{$\boldsymbol{P}$} \\
\cline { 2 - 6 } & \# FP & \# FN & $\begin{array}{c}{ }^{14} \mathbf{C}-U B T \\
{[\%(\mathbf{n} / \mathbf{N})]}\end{array}$ & & & $\begin{array}{c}\text { Discordance } \\
{[\%(\mathbf{n} / \mathbf{N})]}\end{array}$ & \\
\hline $18-30$ & 10 & 0 & $29.4(10 / 34)$ & 3 & 0 & $8.3(3 / 36)$ & 0.032 \\
$31-50$ & 15 & 4 & $14.2(19 / 134)$ & 12 & 4 & $10.2(16 / 157)$ & 0.367 \\
$>50$ & 3 & 3 & $12.2(6 / 49)$ & 19 & 2 & $28.4(21 / 74)$ & 0.045 \\
\hline
\end{tabular}

209 FP: false positive; FN: false negative. The distribution of discordant findings between both UBT

210 assays in each age group was tested using the Fisher's exact test, with a $P$-value less than 0.05

211 considered as statistically significant.

\section{ROC analysis and development of optimal cutoff values}

215 Using a ROC analysis, the optimal cutoff value as a positive indicator for $H$. pylori infection was

$21610.4 \%$ DOB for ${ }^{13} \mathrm{C}-U B T$, and 238 DPM for ${ }^{14} \mathrm{C}$-UBT (Figure 2). With these cutoffs, the AUCs

217 for ${ }^{13} \mathrm{C}$ - and ${ }^{14} \mathrm{C}$-UBT were $86.4 \%$ and $87.8 \%$, respectively. While the increase in cutoff value

218 had greatly reduced the number of false positives in each UBT assay, improving the specificity

219 from $62.2 \%$ (CI: $50.1 \%-73.0 \%$ ) to $81.1 \%$ (CI:70.0\%-88.9\%) for ${ }^{13} \mathrm{C}-\mathrm{UBT}$, and from $54.7 \%$ (CI:

$22042.8 \%-66.1 \%$ ) to $84 \%$ (CI:73.3\%-91.1\%) for ${ }^{14} \mathrm{C}-\mathrm{UBT}$, the sensitivities decreased to $83.9 \%$

221 (CI:76.6\%-89.3\%) and 82.3\% (CI:76.0\%-87.3\%) (Table 3). 

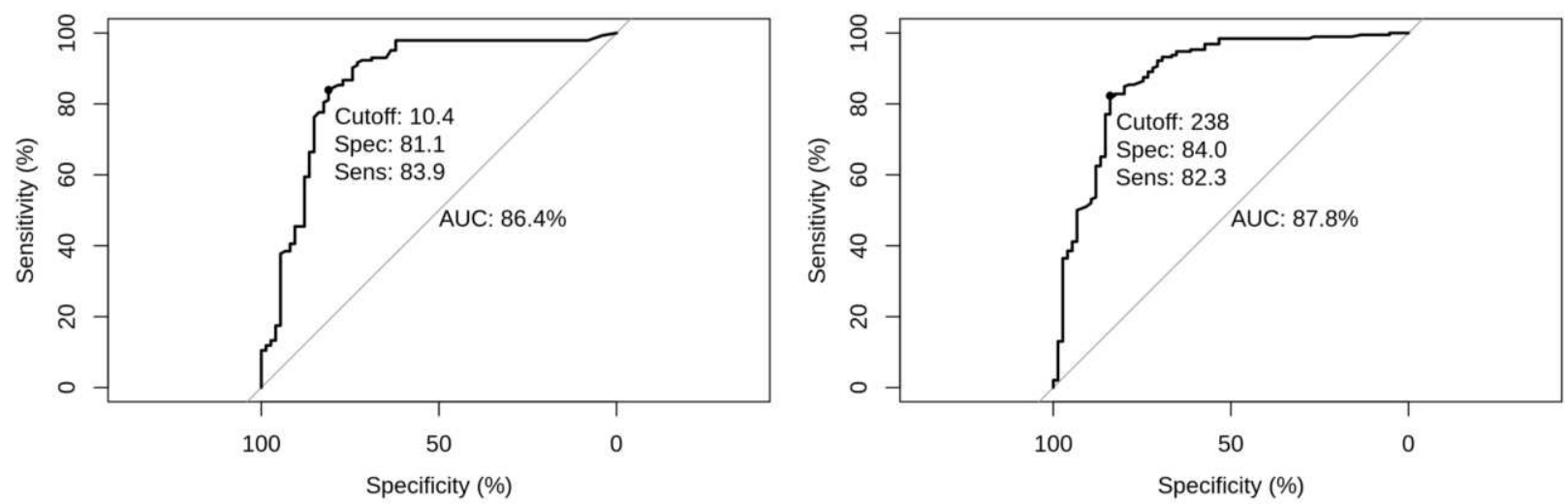

228 Figure 2. ROC curve of UBT for the diagnosis of $H$. pylori infection.

(A) ROC curve of ${ }^{13} \mathrm{C}$-UBT. (B) ROC curve of ${ }^{14} \mathrm{C}$-UBT.

Table 3. Diagnostic performance of the ${ }^{13} \mathrm{C}$-UBT and ${ }^{14} \mathrm{C}$-UBT with optimal cutoff values for $H$.

232 pylori positivity.

\begin{tabular}{ccccccccc} 
Method & \multicolumn{2}{c}{ Histology } & & Sens (\%) & Spec (\%) & Acc (\%) & FPR (\%) & FNR (\%) \\
\cline { 2 - 4 } & & + & - & $95 \% \mathrm{Cl}$ & $95 \% \mathrm{Cl}$ & $95 \% \mathrm{Cl}$ & $95 \% \mathrm{Cl}$ & $95 \% \mathrm{Cl}$ \\
\hline${ }^{13} \mathrm{C}-$-UBT & + & 120 & 14 & 83.9 & 81.1 & 82.9 & 18.9 & 16.1 \\
& - & 23 & 60 & $76.6-89.3$ & $70.0-88.9$ & $77.4-87.4$ & $11.1-30.0$ & $10.7-23.4$ \\
\hline${ }^{14} \mathrm{C}-U B T$ & + & 158 & 12 & 82.3 & 84 & 82.8 & 16 & 17.7 \\
& - & 34 & 63 & $76.0-87.3$ & $73.3-91.1$ & $77.8-86.9$ & $8.9-26.7$ & $12.7-24.0$
\end{tabular}

233 UBT: urea breath test; Sens: sensitivity; Spec: specificity; Acc: accuracy; FPR: false positive

234 rate; FNR: false negative rate; CI: confidence interval. Based on the maximum Youden index

235 method, the optimal cutoff values for $H$. pylori positivity were 10.4\% DOB and 238 DPM for the

$236{ }^{13} \mathrm{C}$-UBT and ${ }^{14} \mathrm{C}$-UBT, respectively.

238 To improve the sensitivity of each assay, we thought that it was necessary to establish an

239 additional cutoff value as a negative indicator of $H$. pylori infection, following which the values

240 situated between the upper and lower cutoffs would be classified as indeterminate results and

241 therefore require repeated testing. Again, ROC analysis was performed on each UBT assay and 
242 this time, only with values below the optimal cutoff as previously determined. As shown in

243 Figure 3, the new cutoff value was 3\% DOB for ${ }^{13} \mathrm{C}-\mathrm{UBT}$, and 87 DPM for ${ }^{14} \mathrm{C}-\mathrm{UBT}$, which in

244 turn implies that UBT values less than 3\% DOB or 87 DPM were very likely to be H. pylori

245 negative. Taking both upper and lower cutoff values for each assay into consideration, we

246 recommend that for ${ }^{13} \mathrm{C}$-UBT readings of $3 \%$ to $10.3 \%$ and ${ }^{14} \mathrm{C}$-UBT readings of 87 to 237 , the

247 H. pylori infection status should be considered indeterminate and would therefore require a

248 repeated testing.

249

A

B

250

251

252

253
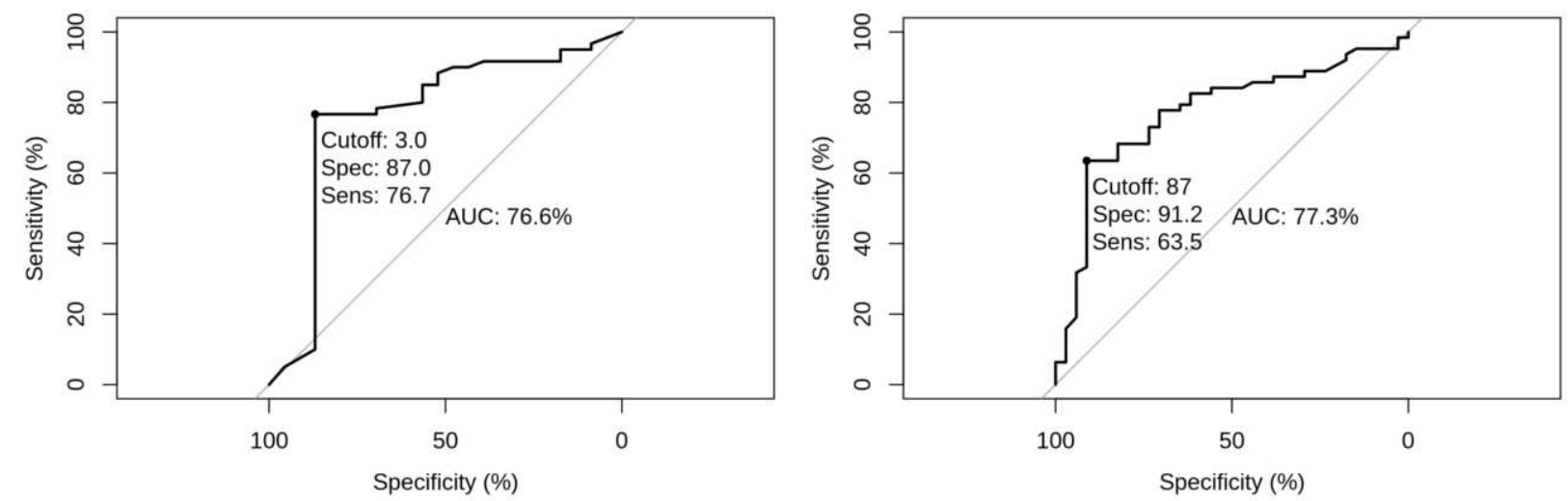

254

255 Figure 3. ROC curve of UBT for the diagnosis of $\mathrm{H}$. pylori infection with only UBT readings 256 below the optimal positive cutoff value.

257 (A) ROC curve of ${ }^{13} \mathrm{C}-U B T$. (B) ROC curve of ${ }^{14} \mathrm{C}-\mathrm{UBT}$.

258

259 More importantly, with the introduction of an indeterminate zone, in which its (indeterminate)

260 readings have been excluded from performance assessment and subjected to other test methods,

261 the sensitivity and specificity can be improved to 96\% (CI:90.4\%-98.5\%) and 76.7\% (CI:63.7\%-

$26286.2 \%$ ) for ${ }^{13} \mathrm{C}-\mathrm{UBT}$, and $98.1 \%$ (CI:94.2\%-99.5\%) and 76.9\% (CI:62.8\%-87.0\%) for ${ }^{14} \mathrm{C}-\mathrm{UBT}$ 
263 (Table 4). Further, the diagnostic accuracy of the ${ }^{13} \mathrm{C}$ - and ${ }^{14} \mathrm{C}$-UBT in this population can be

264 boosted from initially $83.9 \%$ (CI:78.4\%-88.2\%) and 85\% (CI:80.2\%-88.8\%), to 89.7\%

265 (CI:84.5\%-93.4\%) and 93\% (CI:88.6\%-95.8\%), respectively.

266

267 Table 4. Diagnostic performance of the ${ }^{13} \mathrm{C}$-UBT and ${ }^{14} \mathrm{C}$-UBT with optimal $H$. pylori-positive 268 and -negative cutoff values, and the inclusion of an indeterminate range.

\begin{tabular}{cccccccccc}
\hline \multirow{2}{*}{ Method } & & Histology & & Sens (\%) & Spec (\%) & Acc (\%) & FPR (\%) & FNR (\%) \\
\cline { 2 - 4 } & & + & - & & $95 \% \mathrm{Cl}$ & $95 \% \mathrm{Cl}$ & $95 \% \mathrm{Cl}$ & $95 \% \mathrm{Cl}$ & $95 \% \mathrm{Cl}$ \\
\hline${ }^{13} \mathrm{C}-$-UBT & + & 120 & 14 & & 96 & 76.7 & 89.7 & 23.3 & 4
\end{tabular}

\begin{tabular}{ccc}
$(\geq 10.4 \%)$ & & \\
\hline- & 5 & 46 \\
$(\leq 2.9 \%)$ & & \\
\hline IND & 18 & 14
\end{tabular}

$90.4-98.5 \quad 63.7-86.2 \quad 84.5-93.4 \quad 13.8-36.3 \quad 1.5-9.6$

$(3-10.3 \%)$

\begin{tabular}{|c|c|c|c|c|c|c|c|c|}
\hline \multirow[t]{6}{*}{${ }^{14} \mathrm{C}$-UBT } & + & 158 & 12 & 98.1 & 76.9 & 93 & 23.1 & 1.9 \\
\hline & ( $\geq 238$ DPM) & & & $94.2-99.5$ & $62.8-87.0$ & $88.6-95.8$ & $13.0-37.2$ & $0.5-5.8$ \\
\hline & - & 3 & 40 & & & & & \\
\hline & ( $\leq 86$ DPM) & & & & & & & \\
\hline & IND & 31 & 23 & & & & & \\
\hline & (87-237 DPM) & & & & & & & \\
\hline
\end{tabular}

269 UBT: urea breath test; IND: indeterminate; Sens: sensitivity; Spec: specificity; Acc: accuracy;

270 FPR: false positive rate; FNR: false negative rate; $\mathrm{CI}$ : confidence interval.

271

272 Discussion

273 The urea breath test is widely accepted as an accurate non-invasive method for diagnosing $H$.

274 pylori infection. In the present study, we assessed the diagnostic performance of the ${ }^{13} \mathrm{C}$ - and ${ }^{14} \mathrm{C}$ - 
275 UBT commercial kits used in our hospital by comparing each UBT outcome against that of 276 histological examination, which was considered the "gold standard" reference method for

277 determination of $H$. pylori infection. Among the 164 and $53{ }^{13} \mathrm{C}$-UBT-positive and -negative

278 patients, only $136(82.9 \%, 136 / 164)$ and $46(86.8 \%, 46 / 53)$ in whom the $H$. pylori infection status

279 was concordant with that of histopathology examination. On the other hand, among the 220 and

$280 \quad 47{ }^{14} \mathrm{C}$-UBT-positive and -negative patients, $186(84.5 \%, 186 / 220)$ and $41(87.2 \%, 41 / 47)$ were

281 positive and negative for $H$. pylori based on histopathology examination, respectively. Using the

282 original cutoff values of $4 \%$ DOB and 100 DPM, both the ${ }^{13} \mathrm{C}$ - and ${ }^{14} \mathrm{C}-\mathrm{UBT}$ assays had high

283 sensitivity, at $95.1 \%$ and $96.9 \%$, respectively, but inadequate specificity, which was $62.2 \%$ for

284 the former and $54.7 \%$ for the latter. Given the unexpectedly high number of false positives

285 produced by each test, we investigated and compared the distribution of discordant UBT and

286 histological findings in different age groups. Importantly, based on histology, the ${ }^{14} \mathrm{C}-\mathrm{UBT}$ was

287 significantly more accurate than the ${ }^{13} \mathrm{C}$-UBT in determining $H$. pylori infection status in patients

288 aged 18-30 years, whereas, in patients older than 50 years of age, the ${ }^{13} \mathrm{C}$-UBT method was more

289 accurate than the ${ }^{14} \mathrm{C}-\mathrm{UBT}$.

290 The ${ }^{13} \mathrm{C}$-UBT measures the ratio of labeled $\mathrm{CO}_{2}$ to human respiratory $\mathrm{CO}_{2}$ in the breath. Hence

291 its outcome can be affected by one's gender, age, urea hydrolysis rate and $\mathrm{CO}_{2}$ production rate

292 (15). Therefore, in the event where there were many false-positive ${ }^{13} \mathrm{C}$-UBT results among the

293 young patients, it is possible that these individuals have a relatively low basal $\mathrm{CO}_{2}$ production

294 rate and/or a high urea hydrolysis rate, releasing breath with a proportionally higher quantity of 
295 labeled $\mathrm{CO}_{2}$ and thus, generating a false-positive $\mathrm{DOB}$ value. In the situation where ${ }^{14} \mathrm{C}-\mathrm{UBT}$

296 generated substantially more false-positive results than ${ }^{13} \mathrm{C}$-UBT in older patients, some of these

297 patients might have hypochlorhydria, a condition where there is a low-level production of gastric

298 acid and which is commonly associated with aging, leading to the growth of urease-producing

299 non-H. pylori bacteria originating either from the oral cavity or the intestine and thus, a UBT-

300 positive outcome (16-18).

301 Also, it is also possible that the four-hour fasting time (prior to ingesting a capsule containing 50

$302 \mathrm{mg}{ }^{13} \mathrm{C}$ isotope-labeled urea) of this current study is insufficient to empty the stomach in some of

303 these individuals. This situation is most likely to generate a less-acidic gastric environment,

304 which would be rather permissive for the growth of other bacteria with urease activity that could

305 eventually induce a false-positive UBT reaction. Therefore, a longer fasting period, potentially

306 overnight when possible, should be considered the preferred option before testing.

307 Additionally, attention to detail when performing the tests could improve the accuracy. As an

308 example, cleaning teeth and mouth immediately prior to the test might decrease gastric

309 contamination from swallowed oropharyngeal (urease positive) bacteria. Ensuring the patient was

310 sitting quietly prior to the test would lower the amount of endogenous $\mathrm{CO}_{2}$ resulting in a slightly

311 higher breath enrichment of the isotope.

312 Depending on populations and the doses of ${ }^{13} \mathrm{C}$-urea or ${ }^{14} \mathrm{C}$-urea, no one-size-fits-all UBT cutoff

313 value can be used to define whether an individual is $H$. pylori-positive or -negative (19-22). In

314 our study, to overcome the low specificity of each UBT kit, two optimal cutoff points, indicating 
315 UBT-positive and -negative, respectively, along with an indeterminate zone to address UBT

316 readings that are inconclusive, were established. The intermediate zone, defined as ranging from

$3173 \%$ to $10.3 \%$ DOB for ${ }^{13} \mathrm{C}-\mathrm{UBT}$, and from $87-237 \mathrm{DPM}$ for ${ }^{14} \mathrm{C}-\mathrm{UBT}$, contained at least half of

318 the false-positive test results in this study. By using new optimal cutoff values and including an

319 indeterminate range, the false positive rates can be greatly reduced. More importantly, we suggest

320 that for any patient who had an indeterminate UBT result, a repeated UBT or other diagnostic test

321 such as stool antigen or serum antibody test should be performed to confirm $H$. pylori infection,

322 avoiding misdiagnosis and unnecessary antibiotic treatment.

\section{Limitations of study}

324 We concede that there are limitations in this study which was performed in a busy clinical setting

325 rather than in a formal research environment. Despite with the new cutoffs and the establishment

326 of an indeterminate range, the specificity of each UBT kit was only improved to approximately

$32777 \%$, which is still considerably lower than reported (10). The lower specificity of each UBT kit

328 used in this study was probably due to the misdiagnosis of $H$. pylori infection when histology

329 alone was used as the reference method, as its accuracy depends on the skills of the operator, the

330 size and number of biopsies taken and whether or not the biopsy site contained H. pylori or

331 missed it by chance.

332 To address this issue, a larger cohort study with a more even distribution of different age

333 categories, as well as the ability to test every study participant using both ${ }^{13} \mathrm{C}$ - and ${ }^{14} \mathrm{C}$-UBT, and

334 the concordant use of two or three methods as references should be further performed. For 
335 example, rapid urease test, bacterial culture, histology and even PCR, would create a truer "gold

336 standard" and allow for better comparison of the diagnostic accuracy of both tests and the

337 validation of our suggested indeterminate zone. At the same time, observation of how the tests

338 were administered in a busy clinical setting could increase the value of the test even further.

\section{Conclusions}

340 Via comparing the UBT outcomes to that of histopathology examination, we demonstrated that

341 both ${ }^{13} \mathrm{C}$ - and ${ }^{14} \mathrm{C}$-UBT kits used in this study have high sensitivity but low specificity. Based on

342 ROC analysis and the maximum Youden index method, new optimal cutoff values were

343 identified and used to establish an indeterminate range (3-10.3\% DOB for ${ }^{13} \mathrm{C}-\mathrm{UBT}$ and $87-237$

344 DPM for ${ }^{14} \mathrm{C}$-UBT), improving the specificity from $62.2 \%$ to $76.7 \%$ and $54.7 \%$ to $76.9 \%$ for the

$345{ }^{13} \mathrm{C}$ - and ${ }^{14} \mathrm{C}$-UBT, respectively. We strongly suggest that for any patient who had an

346 indeterminate UBT result, a repeated UBT or other diagnostic test should be performed to

347 confirm $H$. pylori infection, avoiding misdiagnosis and unnecessary antibiotic treatment. For

348 future studies, a larger cohort study with two or three methods as references should be further

349 performed to validate our suggested indeterminate zone.

351 Declarations

352 Ethics approval and consent to participate 
353 This study was approved by the research ethics committee of Shenzhen Kuichong People's

354 Hospital (reference no. 201609) and registered at www.chictr.org.cn (reference no.

355 ChiCTR2000041570). Informed consents were obtained from all participants.

\section{Consent for publication}

357 Not applicable.

\section{Availability of data and materials}

359 All data generated or analysed during this study are included in this published article (and its

360 supplementary information files).

\section{Competing interests}

362 The authors declare that they have no competing interests.

\section{Funding}

364 This work was funded by the National Natural Science Foundation of China (No. 81902022) and

365 the Sanming Project of Medicine in Shenzhen (No. SZSM201510050).

\section{Authors' contributions}

367 Preparation of draft manuscript: XY and SZ. Manuscript revision: EGC, MJW and CYT. Data

368 analysis: EGC, MJW and CYT. Endoscopy: YH, AL, DS, The urea breath test and

369 histopathology test: SZ, XL, HC and DS. Study design and conceptualisation: CYT, BJM and

370 XL. Funding and resources: XY, YH. All authors read and approved the final manuscript.

\section{Acknowledgements}

372 Not applicable. 


\section{Authors' information}

$374{ }^{1}$ Department of Gastroenterology, Shenzhen Second People's Hospital/ the First Affiliated

375 Hospital of Shenzhen University, Health Science Center, Shenzhen 518035, China.

$3762^{2}$ Department of Clinical Laboratory, Kuichong People's Hospital, Shenzhen 518116, China.

$377{ }^{3}$ The Marshall Centre for Infectious Diseases Research and Training, University of Western

378 Australia, Perth 6009, Australia.

$379{ }^{4}$ Department of Gastroenterology, Kuichong People's Hospital, Shenzhen 518116, China.

$380{ }^{5}$ Department of Nuclear Medicine, Shenzhen People's Hospital, Shenzhen 518001, China.

$381{ }^{6}$ Department of Computer Science and Software Engineering, University of Western Australia,

382 Perth 6009, Australia.

383

384 References

385 1. Hooi JKY, Lai WY, Ng WK, Suen MMY, Underwood FE, Tanyingoh D, et al. Global Prevalence of

386 Helicobacter pylori Infection: Systematic Review and Meta-Analysis. Gastroenterology. 2017;153(2):420-9.

387 2. Marshall BJ, Warren JR. Unidentified curved bacilli in the stomach of patients with gastritis and peptic

388 ulceration. Lancet. 1984;1(8390):1311-5.

389 3. Marshall BJ, Windsor HM. The relation of Helicobacter pylori to gastric adenocarcinoma and

390 lymphoma: pathophysiology, epidemiology, screening, clinical presentation, treatment, and prevention.

391 Med Clin North Am. 2005;89(2):313-44, viii.

392 4. Bauerfeind P, Garner R, Dunn BE, Mobley HL. Synthesis and activity of Helicobacter pylori urease and 
393 catalase at low pH. Gut. 1997;40(1):25-30.

394 5. Savarino V, Vigneri S, Celle G. The 13C urea breath test in the diagnosis of Helicobacter pylori infection.

395 Gut. 1999;45 Suppl 1:I18-22.

396 6. Hamlet AK, Erlandsson KI, Olbe L, Svennerholm AM, Backman VE, Pettersson AB. A simple, rapid,

397 and highly reliable capsule-based 14C urea breath test for diagnosis of Helicobacter pylori infection. Scand

398 J Gastroenterol. 1995;30(11):1058-63.

399 7. Stubbs JB, Marshall BJ. Radiation dose estimates for the carbon-14-labeled urea breath test. J Nucl Med.

400 1993;34(5):821-5.

401 8. Bentur Y, Matsui D, Koren G. Safety of 14C-UBT for diagnosis of Helicobacter pylori infection in

402 pregnancy. Canadian family physician Medecin de famille canadien. 2009;55(5):479-80.

403 9. Negida A, Fahim NK, Negida Y. Sample Size Calculation Guide - Part 4: How to Calculate the Sample

404 Size for a Diagnostic Test Accuracy Study based on Sensitivity, Specificity, and the Area Under the ROC

405 Curve. Advanced journal of emergency medicine. 2019;3(3):e33.

406 10. Ferwana M, Abdulmajeed I, Alhajiahmed A, Madani W, Firwana B, Hasan R, et al. Accuracy of urea

407 breath test in Helicobacter pylori infection: meta-analysis. World journal of gastroenterology.

$408 \quad 2015 ; 21(4): 1305-14$.

409 11. Yang B, Chen H, Li S. Analysis of helicobacter pylori infection in Longgang distric of Shenzhen.

410 Proceeding of Clinical Medicine. 2016;25(11):816-7+74.

411 12. Zweig MH, Campbell G. Receiver-operating characteristic (ROC) plots: a fundamental evaluation tool

412 in clinical medicine. Clinical chemistry. 1993;39(4):561-77. 
413 13. Robin X, Turck N, Hainard A, Tiberti N, Lisacek F, Sanchez JC, et al. pROC: an open-source package

414 for R and S+ to analyze and compare ROC curves. BMC Bioinformatics. 2011;12:77.

415 14. Youden WJ. Index for rating diagnostic tests. Cancer. 1950;3(1):32-5.

416 15. Klein PD, Malaty HM, Czinn SJ, Emmons SC, Martin RF, Graham DY. Normalizing results of 13C-urea

417 breath testing for CO2 production rates in children. J Pediatr Gastroenterol Nutr. 1999;29(3):297-301.

418 16. Osaki T, Mabe K, Hanawa T, Kamiya S. Urease-positive bacteria in the stomach induce a false-positive

419 reaction in a urea breath test for diagnosis of Helicobacter pylori infection. J Med Microbiol. 2008;57(Pt

420 7):814-9.

421 17. Michaud L, Gottrand F, Ganga-Zandzou PS, Wizla-Derambure N, Turck D, Vincent P. Gastric bacterial

422 overgrowth is a cause of false positive diagnosis of Helicobacter pylori infection using $13 \mathrm{C}$ urea breath test.

423 Gut. 1998;42(4):594-5.

424 18. Brandi G, Biavati B, Calabrese C, Granata M, Nannetti A, Mattarelli P, et al. Urease-positive bacteria

425 other than Helicobacter pylori in human gastric juice and mucosa. Am J Gastroenterol. 2006;101(8):1756-61.

426 19. Zhou Q, Li L, Ai Y, Pan Z, Guo M, Han J. Diagnostic accuracy of the (14)C-urea breath test in

427 Helicobacter pylori infections: a meta-analysis. Wien Klin Wochenschr. 2017;129(1-2):38-45.

428 20. Kato C, Sugiyama T, Sato K, Saito S, Kudara N, Abiko Y, et al. Appropriate cut-off value of 13C-urea

429 breath test after eradication of Helicobacter pylori infection in Japan. J Gastroenterol Hepatol.

$430 \quad 2003 ; 18(12): 1379-83$.

431 21. Mauro M, Radovic V, Zhou P, Wolfe M, Kamath M, Bercik P, et al. 13C urea breath test for (Helicobacter

432 pylori): determination of the optimal cut-off point in a Canadian community population. Can J 
433 Gastroenterol. 2006;20(12):770-4.

434 22. Campuzano-Maya G. An optimized 13C-urea breath test for the diagnosis of H pylori infection. World 435 J Gastroenterol. 2007;13(41):5454-64.

436 


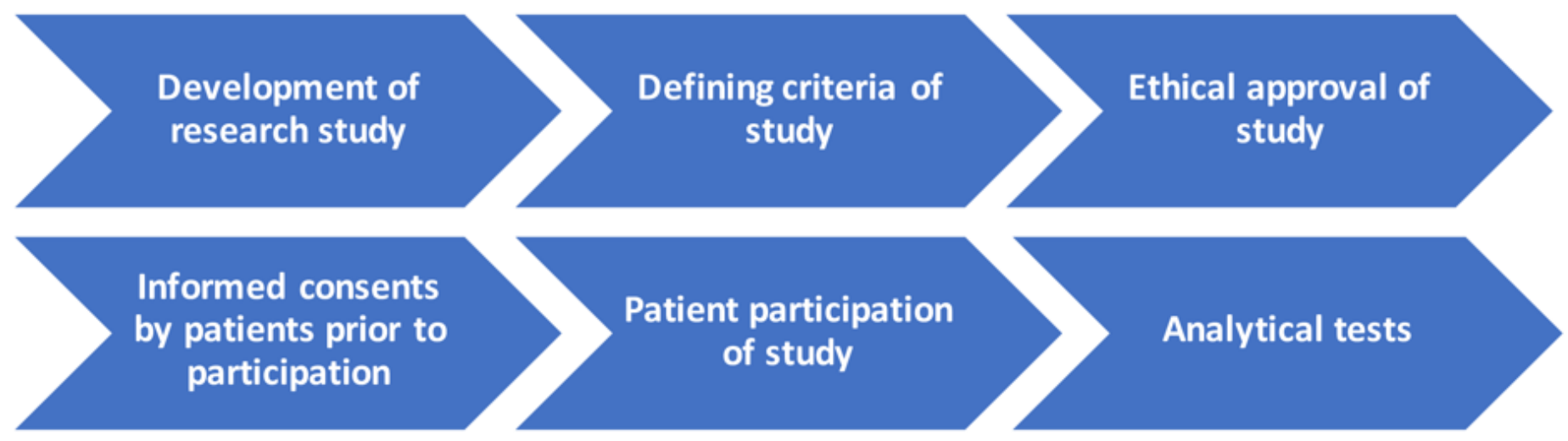

\section{Figure 1}

The overview of entire study.

A

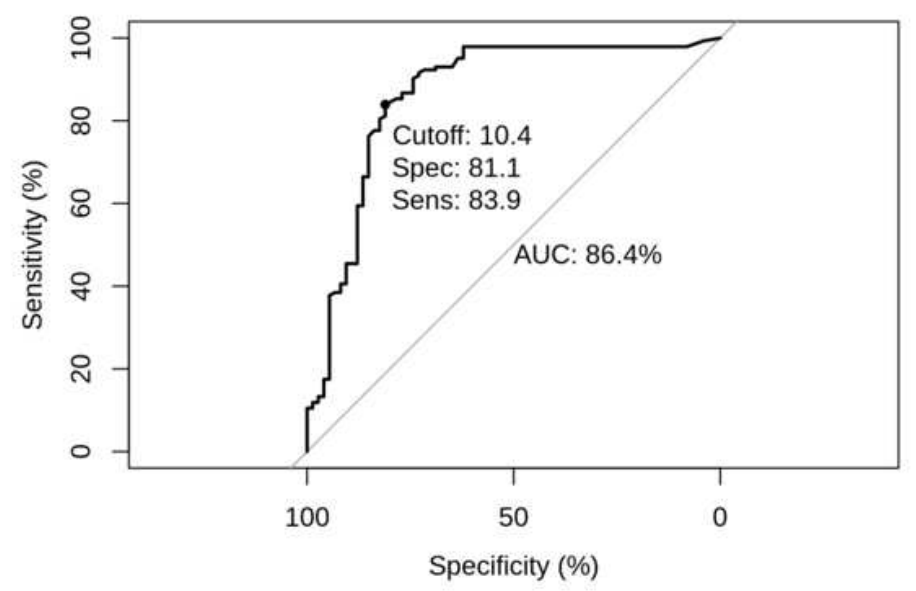

B

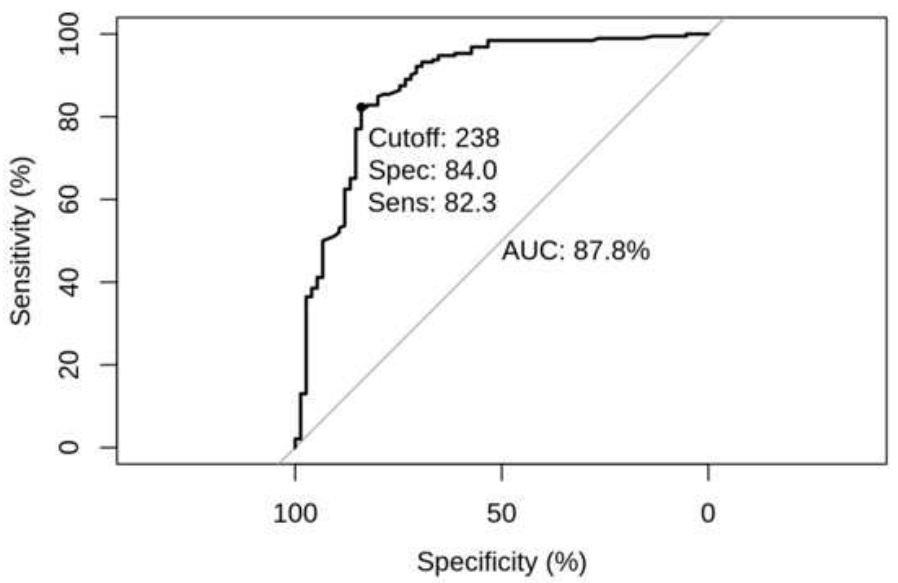

Figure 2

ROC curve of UBT for the diagnosis of H. pylori infection. (A) ROC curve of 13C-UBT. (B) ROC curve of 14C-UBT. 
A

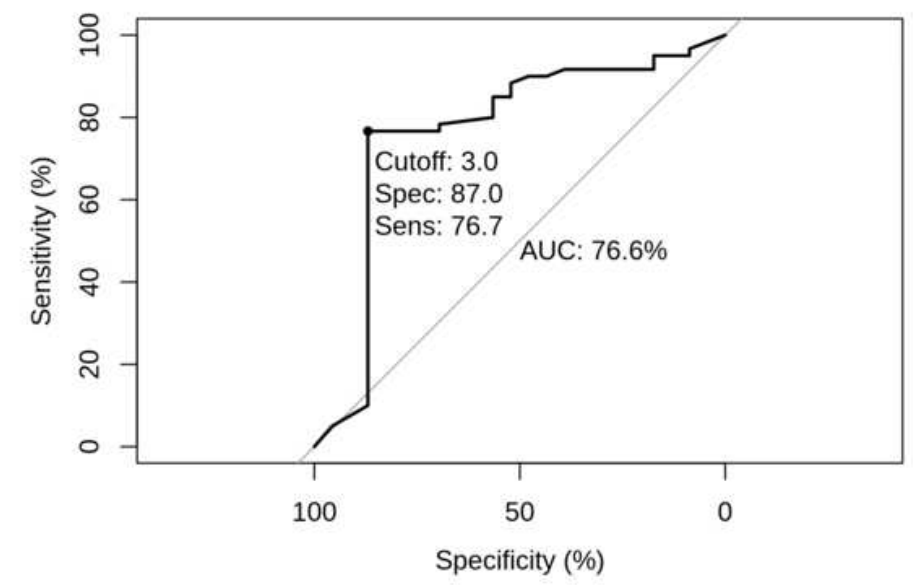

B

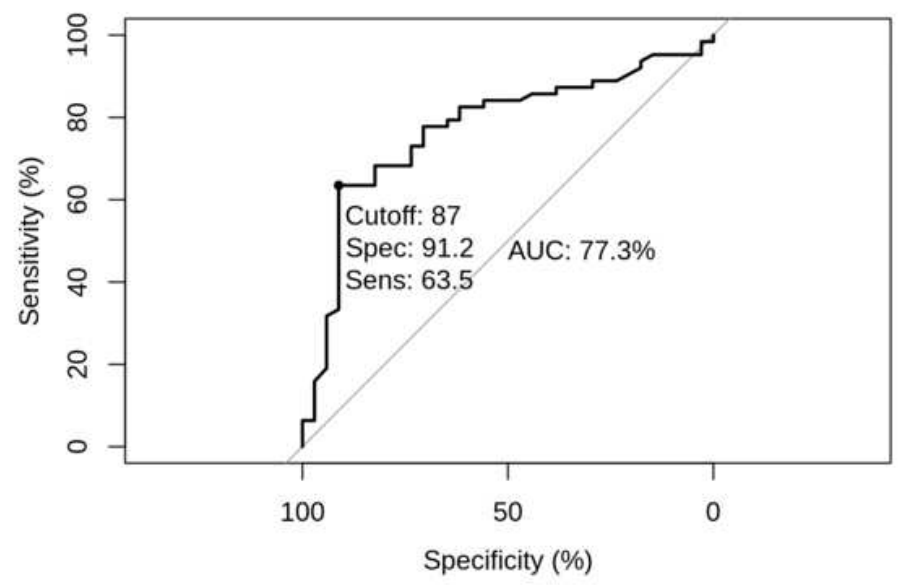

Figure 3

ROC curve of UBT for the diagnosis of $\mathrm{H}$. pylori infection with only UBT readings below the optimal positive cutoff value. (A) ROC curve of 13C-UBT. (B) ROC curve of 14C-UBT.

\section{Supplementary Files}

This is a list of supplementary files associated with this preprint. Click to download.

- TableS1.xIsx 